\title{
THE PRELIMINARY STUDY OF HUANGQI SANYAN DECOCTION BONE-TARGETED LIPOSOME AND IN VITRO EVALUATION OF BONE TARGETING
}

\author{
JIANHONG ZHU \\ and ZHIKUN ZHOU*
}

Department of Pharmacy, Guangdong Medical University, Dongguan, Guangdong 523808, China

\begin{abstract}
In this study, we explored and optimized the preparation process of bone-targeting liposomes from the effective components of Huangqi Sanyan decoction (Astragaloside IV, Icariin, and Notoginsenoside R1) and set up a method that can simultaneous determine Astragaloside IV, Icariin, and Notoginsenoside R1. The physicochemical properties, stability, and bone targeting in vitro of the liposome are evaluated. According to the proportion of clinical combined use, we encapsulated three effective components of Astragalus Sanxian Decoction in liposome by thin-film dispersion method and prepared the bone targeting liposome. And an HPLC method for the simultaneous determination of Astragaloside IV, Icariin, and Notoginsenoside R1 was established. The optimal formulation and preparation process of the liposome is determined as follows: lecithin $100 \mathrm{mg}$, cholesterol $10 \mathrm{mg}$, sodium cholate $10 \mathrm{mg}$, Astragaloside IV $6 \mathrm{mg}$, Icariin $3 \mathrm{mg}$, Notoginsenoside R1 $3 \mathrm{mg}$, E-DTMPA $2 \mathrm{mg}$, organic solvent $10 \mathrm{~mL}$, hydration $30 \mathrm{~min}$, ultrasound $6 \mathrm{~min}$. And the in vitro adsorption test of hydroxyapatite showed that the bone targeting of the EDTA ultrasonic liposome which contains the effective component of Astragalus Sanxian Decoction had significantly enhanced.
\end{abstract}

Keywords: Liposome, bone targeting, Huangqi Sanyan decoction, Astragaloside IV, Icariin, Notoginsenoside R1

Huangqi Sanyan decoction is a classic tonic and invigorating blood circulation formula. Through more than a decade of clinical research, it has been found that Huangqi Sanyan decoction can achieve certain efficacy in the treatment of postmenopausal osteoporosis, and the results of studies have shown that this formula has the advantages of improving the score of renal deficiency symptoms in patients, increasing serum estradiol (E2) levels, increasing bone mineral density, and inhibiting bone resorption in postmenopausal women (1). Animal studies have found that Huangqi Sanyan decoction can increase the content of calcium and phosphorus in the femur of ovariectomized rats, and the bone volume in the upper tibia has a tendency to increase (2). At the same time, it also showed protective effects on the lesions of central nerves in ovariectomized rats. Single herbs such as Radix Astragali, Herba Epimedii, and Radix Notoginseng in Huangqi Sanyan Decoction were proved to have anti-osteoporosis effects. Astragalus membranaceus has the function of inhibiting osteoclasts and also has the potential of enhancing bone formation for a bone supplement (3). Yangbo et al. (4) randomized postmenopausal osteoporotic patients and after 6 months of clinical observation, after treatment with Astragalus membranaceus in the experimental group, the bone mineral density of lumbar spine and femur of patients increased significantly over time, and the blood biochemical parameters improved significantly better than those of the other groups as well, All the results suggest that Astragalus can significantly improve the symptoms of postmenopausal osteoporosis, which can promote bone formation and inhibit bone resorption. Extract of Herba Epimedii significantly increased serum alkaline phosphatase (ALP) activity and inhibited bone loss induced by ovariectomy in ovariectomized rats, while it could significantly increase the rate of bone formation (5). Different concentrations of icariin enhanced the growth and proliferation of osteoblasts, and both icariin concentrations of $100 \mathrm{ng} / \mathrm{mL}$ and $10 \mathrm{ng} / \mathrm{mL}$ also enhanced the

\footnotetext{
\# These authors contributed equally to this work

* Corresponding author: e-mail: zhikunzhou@126.com
} 
secretion of ALP from osteoblasts, indicating that icariin promotes osteoblast proliferation while also enhancing osteoblast osteogenic activity (6). Panax notoginseng has the efficacy of invigorating blood circulation and eliminating pain. Notoginseng can promote osteoblast growth, promote the secretion of IGF-1, and inhibit secretion of IL-6 from osteoblasts, thus inhibiting osteoclast growth and bone resorption to achieve the therapeutic effect of osteoporosis (7), and has some preventive effect on hyperlipidemia induced osteoporosis in rats (8). Shenghuagang et al. (8) explored the effects of group allocation of PNS and Icariin on the proliferation and calcification of osteoblasts cultured in vitro. When the ratio of PNS and icariin components was $5: 2$, it could obviously promote the proliferation of osteoblasts and have obvious calcification, which confirmed the rationality of the combination of PNS and icariin components for the treatment of osteoporosis.

Liposomes, as a drug delivery system, have the advantages of reduced drug toxicity, targeting, slow-release, stability (10-14). The combination of modern liposomal formulation technology and traditional Chinese medicine started in the 1980s, and its properties such as improved efficacy, reduced toxicity, and targeting, sustained release, and cell affinity has been well recognized $(15,16)$, but there are also some problems associated with the study of liposomes in traditional Chinese medicine $(17,18)$. The study of liposomes in traditional Chinese medicine is dominated by that of liposomes for single active pharmaceutical ingredients, in essence, there is no difference from the research on liposome of Western medicines, and only liposomes of multiple components of traditional Chinese medicine can reflect the characteristics of traditional Chinese medicine and also conform to the medication characteristics of traditional Chinese medicine. Some liposomes of traditional Chinese medicine have low drug loading capacity and poor stability, which need to be further addressed.

Hydroxyapatite (HA), the main component of bone, has a molecular formula of $\left[\mathrm{Ca}_{10}\left(\mathrm{Po}_{4}\right)_{6}\right.$ $\left.(\mathrm{OH})_{2}\right]$ and accounts for $84 \%$ of the components of bone salts, and in 1986, pierce (19) first proposed the concept of "bone targeting": compound molecules have a tendency to deposit in bone and incorporate into hydroxyapatite, and Homo osteocalcin has the ability to bind. At present, representative bone targeting drugs are tetracyclines, bisphosphonates, diamine tetramethylbiphosphates, small molecule heterocycles, and so on $(20,21)$.

EDTMPA has a strong affinity for bone tissue and can quickly accumulate around bone tissue, and Guo Jia et al. (22) reported that flexible nanoliposome modified with EDTMPA were stable and achieved $80 \%$ encapsulation efficiency, while also showing excellent bone targeting, with an overall targeting efficiency that was $653.02 \%$ higher than that of plain flexible nanoliposome.

\section{Material}

Astragaloside IV, Icariin, Notoginsenoside R1 were extracted by Baoji Fangsheng Co., Ltd. Glucose gel G50 was obtained from Beijing Soleboard Technology Co., Ltd. Lecithin was produced by ADM, USA. Cholesterol and sodium cholate were purchased from Anhui Tianqi Chemical Technology Co., Ltd. Sucrose was from MP Biomedicals Biomedical Co., Ltd, USA. Hydroxyapatite was obtained from Sinopharm Chemical Reagent Co., Ltd. Ethylenediamine tetramethylene phosphate (E-DTMPA) was purchased from Tokyo Chemical Industry Co., Ltd. Microporous filter membrane was obtained from Tianjin Jinteng Experimental Equipment Co., Ltd. Chromatographic acetonitrile was made by Guangdong Guanghua Science and Technology Co., Ltd. Other agents were of analytical grade from Guangzhou Chemical Reagent Factory.

\section{Determination of active ingredients in Huangqi Sanyan decoction}

Mixed control solutions of the three drugs were assayed using HPLC. The mobile phase is as follows: phase A is ultrapure water, phase $\mathrm{B}$ is acetonitrile. The gradient elution conditions were as follow: 0-8 $\min (80: 20 ; \mathrm{A}: \mathrm{B}), 8-18 \mathrm{~min}(80 \sim 62: 20 \sim 38)$, $18-27 \mathrm{~min}(62: 38)$. The flow rate was $0.8 \mathrm{~mL} / \mathrm{min}$. The detection wavelength was $203 \mathrm{~nm}$. The column temperature was $25^{\circ} \mathrm{C}$ (Figure 1).

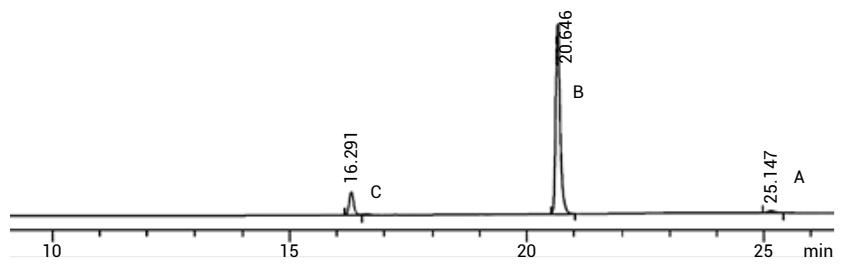

Figure 1. HPLC profiles of Astragaloside IV (A), Icariin (B), Notoginsenoside R1 (C)

\section{Preparation of liposome}

Precisely weighed the recipe quantity of lecithin (PC), cholesterol $(\mathrm{CH})$, Astragaloside IV, Icariin and Notoginsenoside R1 in a round-bottom flask, bath in constant temperature water at $40^{\circ} \mathrm{C}$, then added appropriate amount of methanol chloroform mixed solvent $(1: 1)$ 
and made it fully dissolved, the organic solvent was removed by a rotary evaporator to form a uniform lipid film, and vacuum dried it overnight. Preparing a prescription volume of sodium cholate-PBS solution, added it to the round-bottom flask, and steamed in a constant temperature water bath at $40^{\circ} \mathrm{C}$ in the normal pressure for $30 \mathrm{~min}$. Probe-type ultrasound was used until the initial liposome suspension became translucent. Finally, the primary suspension was squeezed through the microporous membrane in order with $0.80,0.45,0.22 \mu \mathrm{m}$ pore size to obtain the normal ultrasonic liposome (N-UL). In the preparation process, a certain amount of EDTMPA was dissolved in sodium cholate PBS solution, and the above film dispersion method was repeated to prepare EDTMPA liposome ultrasonic liposome (E-UL). It is an EDTMPA modified bone-targeted liposome.

\section{Appearance observation}

The appearance of the two kinds of liposomes was observed and compared, and the suspension of liposomes before and after the formulation optimization (ultrafiltration membrane) was taken on the slide, observed, and photographed under the inverted microscope.

\section{Particle size distribution and entrapment efficiency determination}

The mean particle size diameter of the liposome was directly measured using Zetasizer Nano ZS90 equipment (Malvern Instruments Ltd., Worcestershire, UK). Liposome and unencapsulated free drug were separated by microcolumn centrifugation, the membrane was ruptured by methanol, and the amount of encapsulated drug in liposome was determined by HPLC. Taken liposome was directly membrane disrupted with methanol to measure total drug content.

The entrapment efficiency of drug = amount of drug contained by the formulation / total amount of drug.

\section{Single-factor analysis based on particle size and encapsulation efficiency}

The single factor experiments were used to test the N-UL prepared by film dispersion method, including the effects of lipid concentration, phospholipid to cholesterol mass ratio, hydration time, sonication time, and sodium cholate dosage on N-UL particle size distribution and encapsulation efficiency were analyzed, and the effects of EDTMPA dosage on the particle size distribution and encapsulation efficiency were analyzed.

\section{Long-term stability}

Three batches each of N-UL and E-UL were obtained and stored under natural conditions at room temperature and protected from light at $4^{\circ} \mathrm{C}$, respectively, after nitrogen and absolute oxygen fusion encapsulation, drug entrapment efficiency was determined at 0,1 , and 3 months, and long-term stability of liposome was investigated.

\section{Improvement of liposome stability by freeze-drying}

Two liposome samples were taken separately. One aliquot was added with 5\% sucrose and shaken to dissolve well to obtain a sample of liposomes containing the protecting agent, and both samples were lyophilized. Observations were made on the appearance state and reconstitution process of lyophilized samples, and the changes in appearance change, encapsulation efficiency, and particle size distribution of each liposomal suspension before and after lyophilization were compared.

\section{Evaluation of bone targeting of liposome}

$\mathrm{N}-\mathrm{UL}$ and E-UL were added to the hydroxyapatite (HA) suspension at $37^{\circ} \mathrm{C}$ with constant shaking in a water bath, and one hour later, the suspension from each group was washed through $0.45 \mu \mathrm{m}$ micropore filtration. After methanol ruptured the membrane, HPLC was performed and the peak areas corresponding to Astragaloside IV, Icariin, and Notoginsenoside R1 in each group were recorded to identify the contents of each component from the standard curve (c). Another two sets of liposomal suspensions were taken at $200 \mu \mathrm{L}$ and were measured directly by rupturing the membrane with methanol. The peak areas corresponding to each component were recorded, and the content of each component $(\mathrm{C} 0)$ was identified from the standard curve and the percentage of adsorption was calculated.

\section{RESULT AND DISCUSSION}

\section{Lipid concentration}

With the concentration of lipid increased, the encapsulation efficiency of liposome increased slightly, and the change was no large; the average particle size increased with the increase of concentration, and the change was small (Figure 2, Figure 3).

\section{The mass ratio of $\mathrm{PC}$ and $\mathrm{CH}$}

As the mass ratio of $\mathrm{PC}$ and $\mathrm{CH}$ changed from $3: 1$ to $20: 1$, the encapsulation efficiency of liposomes did not change very much, but the particle 
size decreased significantly. From the results of the single-factor experiment, the smaller mass ratio of $\mathrm{PC}$ and $\mathrm{CH}$ is good for the prescription, but the $\mathrm{CH}$ content is positively correlated with bone targeting (Figure 4, Figure 5).

\section{Hydration time}

The encapsulation efficiency of the liposome increased slightly with the increase of hydration time, but it changed little after more than half an

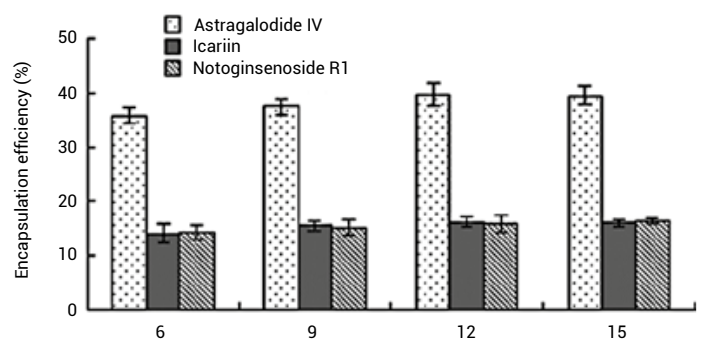

Figure 2. The encapsulation efficiency of different lipid concentration liposomes.

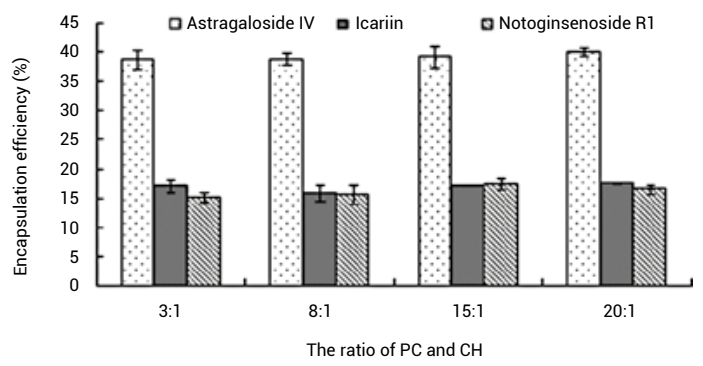

Figure 4. The encapsulation efficiency of different ratios of $\mathrm{PC}$ and $\mathrm{CH}$ liposome.

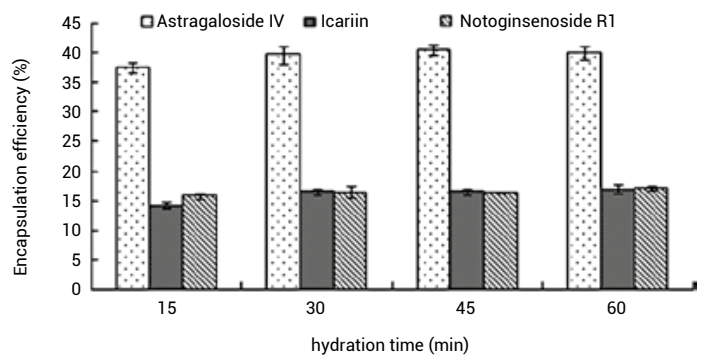

Figure 6. The encapsulation efficiency of different hydration time liposomes.

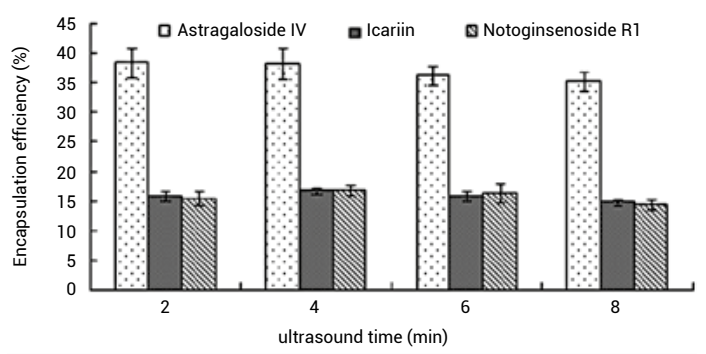

Figure 8 . The encapsulation efficiency of different ultrasound time liposomes. hour and had little effect on the average particle size (Figure 6, Figure 7).

\section{Ultrasound time}

With the increase of ultrasound time to $6 \mathrm{~min}$, the average particle size decreased significantly, and the encapsulation efficiency of liposomes decreased slightly. This indicated that ultrasound time has a certain effect on the basic properties of liposomes (Figure 8, Figure 9).

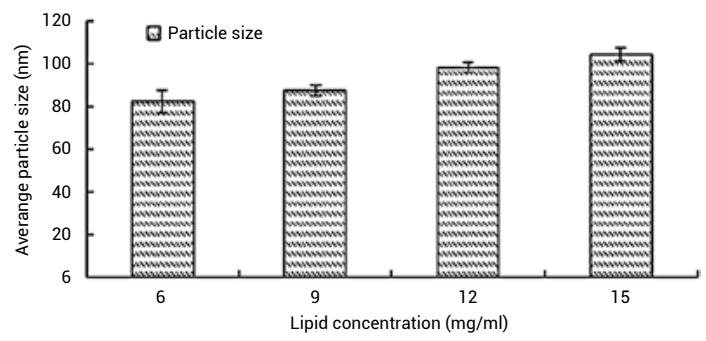

Figure 3. The average particle size of different lipid concentration liposomes.

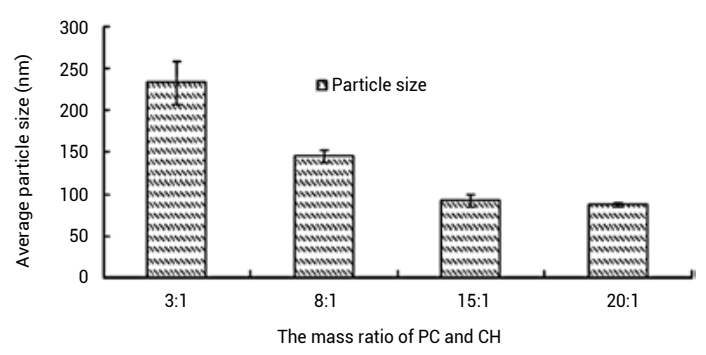

Figure 5. The average particle size of different ratios of $\mathrm{PC}$ and $\mathrm{CH}$ liposome.

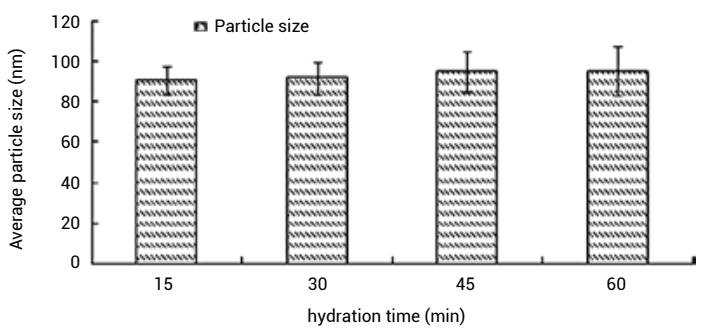

Figure 7. The average particle size of different hydration time liposomes.

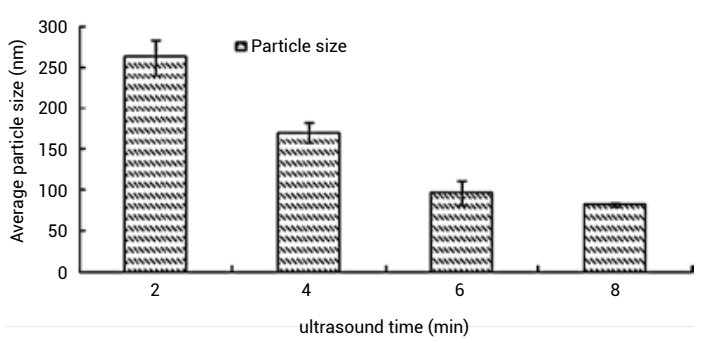

Figure 9. The average particle size of different ultrasound time liposomes. 


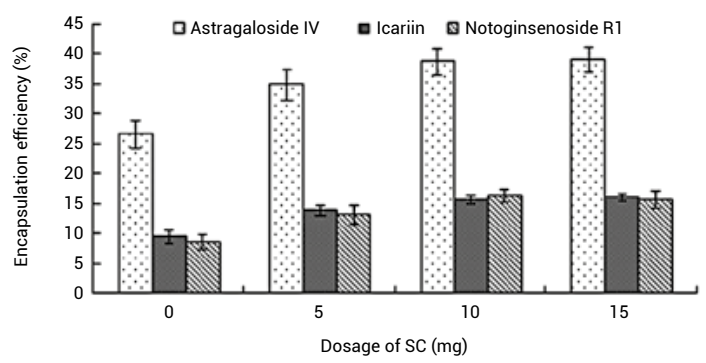

Figure 10. The encapsulation efficiency of different dosages of SC liposome.

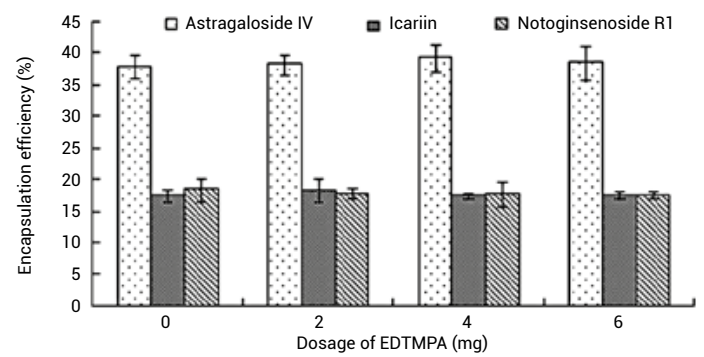

Figure 12. The encapsulation efficiency of different dosages of EDTMPA liposome.

\section{Dosage of sodium cholate}

It can be seen that the amount of sodium cholate had a great influence on the encapsulation efficiency and particle size of the liposome. With the increase of dosage, the encapsulation efficiency of liposomes increased accordingly, and when it increased to $10 \mathrm{mg}$, it tended to be stable basically (Figure 10, Figure 11).

\section{Dosage of EDTMPA}

The change of EDTMPA dosage had little effect on the encapsulation efficiency of liposomes, but the average particle size increased obviously with the increase of EDTMPA dosage (Figure 12, Figure 13).

What preparation method to choose depends on the nature of the drug to be encapsulated, the most important of which is the oil-water partition coefficient $(\log \mathrm{P})$ of the drug (23). Astragaloside IV is water-insoluble and belongs to lipid-soluble drugs (24); Notoginsenoside R1 has a $\log \mathrm{P}$ of 0.034 and belongs to water-soluble drugs (25); The equilibrium solubility of icariin in water at $25^{\circ} \mathrm{C}$ was $15.04 \mathrm{mg} / \mathrm{L}$ with a $\log \mathrm{P}$ of 1.89 (26). Predicted that icariin might be relatively easy to leak from the liposome. And Notoginsenoside R1 is a weak acidic component (27), and the change of $\mathrm{pH}$ may have caused the leakage of the drug. Combining the degree of encapsulation efficiency with the complexity of the experiment, we finally decided to adopt the thinfilm hydration method to prepare liposomes. It was

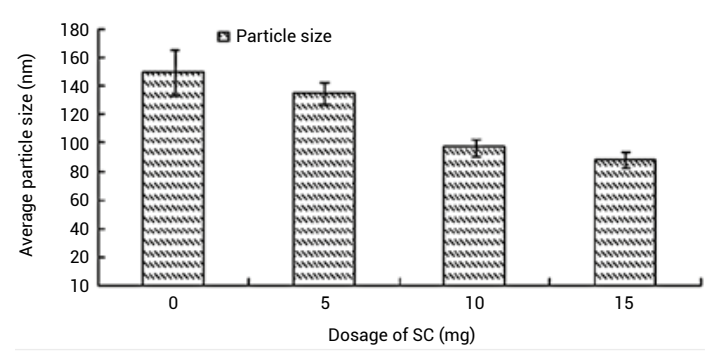

Figure 11. The average particle of different dosages of SC liposome.

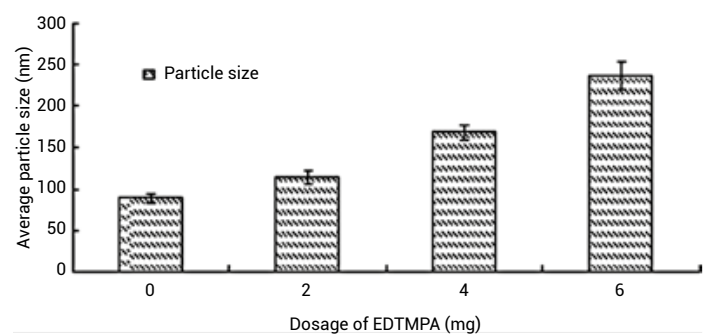

Figure 13. The average particle size of different dosages of EDTMPA liposome.

finally determined that the optimal prescription and preparation process of liposome after modification were as follows: $\mathrm{PC}: \mathrm{CH}: \mathrm{SC}=100: 10: 10$ (mg), Astragaloside IV $6 \mathrm{mg}$, Icariin $3 \mathrm{mg}$, Notoginsenoside R1 $3 \mathrm{mg}$, EDTMPA $2 \mathrm{mg}$, organic solvent $10 \mathrm{~mL}$, hydration time $30 \mathrm{~min}$ and sonication time $6 \mathrm{~min}$.

\section{Physicochemical properties of liposome Morphological observation under a transmission electron microscope}

The microscopic observation revealed that N-UL and E-UL both had a larger particle size before the ultrasound and uneven size distribution E-UL had more needle-like crystals than N-UL, After passing through the ultrasonic filtration membrane, the liposome suspension had a small particle size and no crystal precipitation. (Figure 14, Figure 15)

\section{Determination of particle size distribution and Zeta potential}

The average particle size and zeta potential of the samples were determined by Zetasizer Nano ZS90 equipment. The above data shows that the particle size of the prepared liposome meets the requirements $(n=3)$ (Figure 16, Figure 17, Table 1).

Zeta potential is an important measure of how much charge is involved and an important measure for evaluating the stability of liposomes $(28,29)$. 


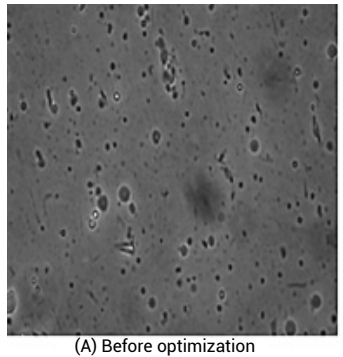

(A) Before optimization

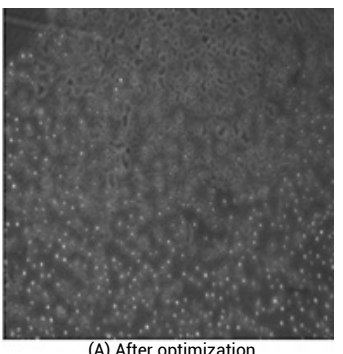

(A) After optimization

Figure 14. The form of N-UL.

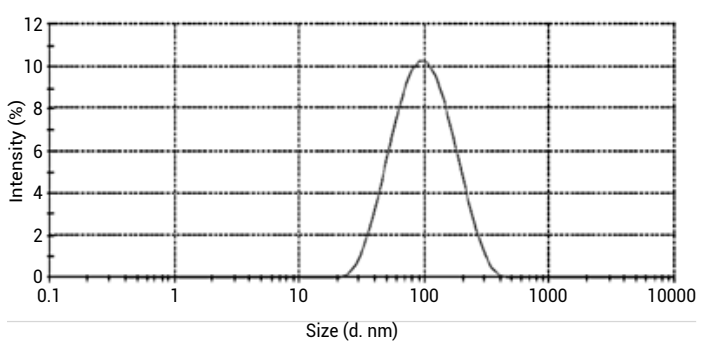

Figure 16. The particle size distribution of N-UL

Membrane material soy lecithin used in this paper to prepare liposome, there are some components such as phosphatidylserine and so on which are electric in phosphate buffer and make the surface of liposome negatively charged, which is beneficial to the stability of liposome.

\section{pH value}

The hydrolysis of lecithin is susceptible to $\mathrm{pH}$ (30), with phospholipids being most stable at $\mathrm{pH}$ 6.5 and having the smallest hydrolysis rate constant. The use of PBS can stabilize the $\mathrm{pH}$ value of the formulation within this range, which is beneficial for improving the stability of liposomes. In this paper, PBS was used as the hydration solvent to prepare liposomes, and the $\mathrm{pH}$ values of the two liposomes

Table 1. Zeta potential values of different liposomes.

\begin{tabular}{|c|c|c|c|}
\hline UL type & blank-UL & N-UL & E-UL \\
\hline Zeta $(\mathrm{mV})$ & -19.10 & -6.73 & -9.81 \\
\hline
\end{tabular}
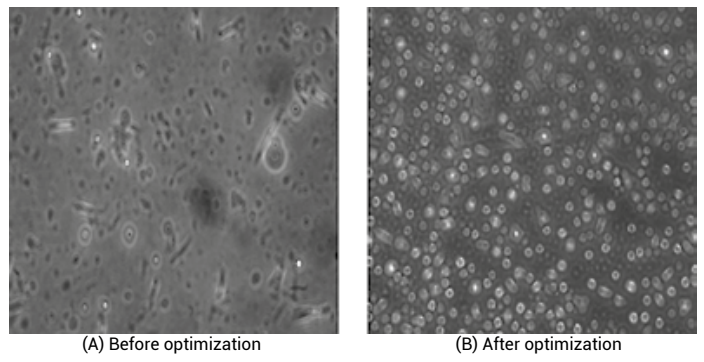

Figure15. The form of E-UL.

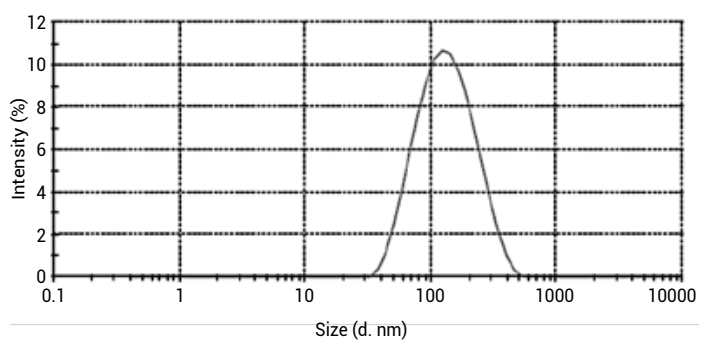

Figure 17. The particle size distribution of E-UL.

were measured to be 6.76 and 6.82 , which met the requirements (Table 2 ).

\section{Stability of liposome \\ Long-term stability}

In the first month, there was no obvious change and difference in the appearance of the two liposomes at room temperature and low temperature. The entrapment efficiency of N-UL and E-UL did not change significantly. In the third month, the appearance of the samples stored at low temperature had no obvious change, but the encapsulation efficiency of N-UL and E-UL decreased. There was a little flocculent at the bottom of the sample placed at room temperature, and the entrapment efficiency decreased significantly (Table 3, Table 4).

Table 2. PH of different liposomes.

\begin{tabular}{|c|c|c|c|}
\hline UL type & blank-UL & N-UL & E-UL \\
\hline $\mathrm{pH}$ & 7.21 & 6.76 & 6.82 \\
\hline
\end{tabular}

Table 3. Long-term stability of N-UL $(n=3)$.

\begin{tabular}{|c|c|c|c|c|c|}
\hline \multirow{2}{*}{$\begin{array}{c}\text { Observation time } \\
\text { (month) }\end{array}$} & Temperature & \multicolumn{3}{|c|}{ Encapsulation efficiency(\%) } & The average particle size \\
\cline { 2 - 6 } & & Astragaloside IV & Icariin & Notoginsenoside R1 & 87.1 \\
\hline 0 & - & 41.7 & 18.4 & 17.5 & 86.4 \\
\hline \multirow{2}{*}{1} & $4^{\circ} \mathrm{C}$ & 40.1 & 17.3 & 17.2 & 88.3 \\
\cline { 2 - 6 } & $25^{\circ} \mathrm{C}$ & 39.6 & 17.4 & 16.1 & 138.7 \\
\hline \multirow{2}{*}{3} & $4^{\circ} \mathrm{C}$ & 26.7 & 10.3 & 8.4 & 167.3 \\
\hline
\end{tabular}


Table 4. Long-term stability of E-UL $(n=3)$.

\begin{tabular}{|c|c|c|c|c|c|}
\hline \multirow{2}{*}{$\begin{array}{c}\text { Observation time } \\
(\text { month) }\end{array}$} & \multirow{2}{*}{ Temperature } & \multicolumn{3}{|c|}{ Encapsulation efficiency (\%) } & The average particle size \\
\cline { 3 - 6 } & & Astragaloside IV & Icariin & Notoginsenoside R1 & 105.4 \\
\hline 0 & - & 40.6 & 18.7 & 18.1 & 17.9 \\
\hline \multirow{2}{*}{1} & $4^{\circ} \mathrm{C}$ & 39.3 & 18.5 & 17.4 & 110.3 \\
\cline { 2 - 6 } & $25^{\circ} \mathrm{C}$ & 38.8 & 18.2 & 10.6 & 147.9 \\
\hline \multirow{2}{*}{3} & $4^{\circ} \mathrm{C}$ & 28.4 & 9.6 & 6.3 & 187.2 \\
\cline { 2 - 6 } & $25^{\circ} \mathrm{C}$ & - & 5.9 & & 106.7 \\
\hline
\end{tabular}

\section{Improvement of liposome stability by freeze-drying}

The lyophilized powder of the two liposomes was milky white and dissolved in PBS. The encapsulation efficiency of N-UL and E-UL without sucrose decreased significantly, and the particle size increased significantly; The entrapment efficiency of $\mathrm{N}-\mathrm{UL}$ and E-UL with 5\% sucrose as protective agent decreased and the particle size increased slightly. The changes in encapsulation efficiency and particle size distribution of the two liposomes with sucrose as a protective agent before and after freeze-drying were smaller than those without a protective agent (Table 5, Table 6).

Sucrose is often used as a lyoprotectant for liposomes, and the retention rate of the entrapment can reach more than $75 \%$ after lyophilization and rehydration of liposomes by lyophilization $(31,32)$. Guo
Jia et al. studied the effect of sucrose on the lyophilization protection of common flexible nanoliposomes and found that $5 \%$ sucrose was used as a protective agent, and the retention rate of the entrapped drugs after liposome reconstitution was nearly $90 \%$ (33). In this paper, $5 \%$ sucrose was selected as the lyoprotectant, and the obtained preparation was stored by lyophilization. It was found that the presence of sucrose prevented the significant increase in liposome particle size after hydration, but did not prevent drug leakage and did not achieve the desired effect. This may be related to the nature of the drug, which is damaged mechanically during lyophilization leading to rupture of the membrane, causing leakage of the drug (34).

\section{Evaluation of bone targeting of liposome}

The results showed that hydroxyapatite has a certain adsorption rate for Astragaloside IV, Icariin,

Table 5. Effect of protectant addition on lyophilized samples of N-UL $(n=3)$.

\begin{tabular}{|c|c|c|c|c|c|}
\hline \multirow{2}{*}{ Protective agent } & \multicolumn{3}{|c|}{ Encapsulation efficiency (\%) } & The average particle size \\
\cline { 2 - 6 } & Astragaloside IV & Icariin & Notoginsenoside R1 & $(\mathrm{nm})$ \\
\hline \multicolumn{2}{|c|}{ Primary liposome suspensions } & 41.7 & 18.4 & 17.5 & 87.1 \\
\hline $\begin{array}{c}\text { Reconstituted } \\
\text { liposome } \\
\text { suspensions }\end{array}$ & Blank group & - & 2.2 & 1.7 & 283.6 \\
\cline { 2 - 6 } & Sucrose & 28.7 & 8.7 & 10.3 & 98.2 \\
\hline
\end{tabular}

Table 6. Effect of protectant addition on lyophilized samples of E-UL $(n=3)$.

\begin{tabular}{|c|c|c|c|c|c|}
\hline \multirow{2}{*}{ Protective agent } & \multicolumn{3}{|c|}{ Encapsulation efficiency (\%) } & The average particle size \\
\cline { 2 - 6 } & Astragaloside IV & Icariin & Notoginsenoside R1 & (nm) \\
\hline Primary liposome suspensions & 40.6 & 18.7 & 18.1 & 105.4 \\
\hline $\begin{array}{c}\text { Reconstituted } \\
\text { liposome } \\
\text { suspensions }\end{array}$ & Blank group & - & 1.9 & 2.8 & 288.3 \\
\cline { 2 - 6 } & Sucrose & 27.1 & 7.4 & 9.5 & 116.4 \\
\hline
\end{tabular}

Table 7. Drug concentration and adsorption rate before and after addition of E-UL to HA.

\begin{tabular}{|c|c|c|c|}
\hline Drug & $\mathrm{C}_{0}(\mathrm{mg} / \mathrm{mL})$ & $\mathrm{C}(\mathrm{mg} / \mathrm{mL})$ & The percentage of adsorption $(\%)$ \\
\hline Astragaloside IV & 0.540 & 0.505 & 5.5 \\
\hline Icariin & 0.241 & 0.227 & 5.8 \\
\hline Notoginsenoside R1 & 0.217 & 0.205 & 5.5 \\
\hline
\end{tabular}


Table 8. Drug concentration and adsorption rate before and after addition of N-UL to HA.

\begin{tabular}{|c|c|c|c|}
\hline Drug & $\mathrm{C}_{0}(\mathrm{mg} / \mathrm{mL})$ & $\mathrm{C}(\mathrm{mg} / \mathrm{mL})$ & The percentage of adsorption (\%) \\
\hline Astragaloside IV & 0.560 & 0.556 & 0.7 \\
\hline Icariin & 0.237 & 0.234 & 1.3 \\
\hline Notoginsenoside R1 & 0.223 & 0.220 & 1.3 \\
\hline
\end{tabular}

and Notoginsenoside R1 in N-UL and E-UL, and the adsorption rate for E-UL is significantly higher than that of N-UL (Table 7, Table 8).

\section{CONCLUSION}

We prepared a flexible liposome composed of the effective components of Huangqi Sanyan decoction, and a bone-targeted liposome composed of the effective components of Huangqi Sanyan Decoction modified with EDTMPA as the bone targeting guide and optimized the prescription and process of both formulations. The basic properties such as entrapment efficiency and size distribution of the two liposomes were examined and the physicochemical properties, stability, and storage conditions of the formulations were investigated and the results showed that all the properties were basically in accordance with the General Pharmaceutical requirements. It provides a reference for future studies on TCM compound formulas.

A preliminary study of the bone targeting of the two liposomes was performed by an in vitro hydroxyapatite adsorption assay, and the results showed that both liposomes had some bone targeting ability, and that the EDTMPA modified liposome had a better bone targeting ability. It lays a certain foundation for future research on bone targeting carriers.

\section{Funding information}

The present study was supported by grants from the National Natural Science Foundation of China (grant no. 81774344).

\section{Conflict of interest}

The authors declare no conflicts of interest.

\section{REFERENCE}

1. Zhou Z.K., Zhu X. Q., Zeng X.X., et al.: Chin. J. Basic Med. Tradit. Chin. Med. 7, 49 (2001).

2. Xu Z.M, Zhou Z.K.: Guide Tradit. Chin. Med. 15, 9 (2009).
3. Lin X.S., Cao S.H., Wang J.: Guide Tradit. Chin. Med. 20, 77 (2008).

4. Yang B., Yang J.: Sichuan Medicine 28, 291 (2007).

5. Nian H., Xu L.L., Ma M.H., et al.: J. Integr. Tradit. Chin. Western Med. 4, 628 (2006).

6. Yu B., Yang J.S., Liu Y., et al.: Bone Normalization 18, 17 (2006).

7. Guo F.: Clinical Res. Tradit. Chin. Med. 3, 20 (2011).

8. Na Q.Q., Xie H.: Chin. J. Clin. Pharmacol. Ther. 15, 277 (2010).

9. Sheng H.G., Li N., Zhu L.Q., et al.: Chin. J. Experimental Prescriptions 18, 183 (2012).

10. Gulati M., Grover M., Singh S., Singh M.: Int. J. Pharm. 165, 129 (1998).

11. Felnerova D., Viret J.F., Glück R., Moser C.: Curr. Opin. Biotechnol. 15, 518 (2004).

12. Mufamadi M.S., Pillay V., Choonara Y.E., Du Toit L.C, Modi G.: et al.: J. Drug Deliv. 2011, 19 pages (2011).

13. Gangwar M., Singh R., Goel R.K., Nath G.: Asian Pac. J. Trop. Biomed. 2, S1176 (2012).

14. Venkataram S., Awni W.M., Jordan K., Rahman Y.E.: J. Pharm. Sci. 79, 216 (1990).

15. Zhong H., Deng Y., Wang X., Yang B.: Int. J. Pharm. 301, 15 (2005).

16. Deng X.K., Cai B.C., Lv X.Y., et al.: CNJ 15, 963 (2006).

17. Qian S., Li C., Zuo Z.: Curr. Drug Metab. 13, 372 (2012).

18. Chen Z.X.: J. Practical Chin. Med. 26, 587 (2010).

19. Pierce W.M., Leonard W.C.: Eur. Pat. 11, 201057 (1986).

20. Wang D., Miller S.C., Kopecková P., Kopecek J.: Adv. Drug Deliv. Rev. 57, 1049 (2005).

21. Zheng H., Huang W.C., Lou R.L., et al.: J. Pharmacy 38, 75 (2003).

22. Guo J., Zhang M., Li Y.F., et al.: J. Peking University 42, 203 (2009).

23. Shun Q.X., Shao W., Huang G.H.: Chin. Patent Medicine 32, 1397 (2010).

24. Liu A.N., Chen H., Tang X.: Chin. J. Pharmaceutics 7, 290 (2009). 
25. Zhai Y.S., Du S.Y., Xu B., et al.: Chin. J. Tradit. Chin. Med. 35, 984 (2010).

26. Wang Y.L., Jia X.B., Chen Y., et al.: Chin. J. Tradit. Chin. Med. 23, 777 (2008).

27. Lai L., Liu H.G., Wen L., et al.: Chin. J. New Drugs 21, 1050 (2012).

28. Grit M., Underberg W.J., Crommelin D.J.: J. Pharm. Sci. 82, 362 (1993).

29. Ruozi B., Tosi G., Leo E., et al.: Talant 73, 12 (2007).
30. Zhou W., Liu W.L., Liu W., et al.: Food Sci. 33, 128 (2012).

31. Tang W.Y., Song Y.Z., Deng Y.H.: J. Shenyang Pharm. Univ. 29, 560 (2012).

32. Ausborn M., Schreier H., Brezesinski G., Fabian H., Meyer H.W., et al.: J. Control. Release 30, 105 (1994).

33. Guo J.: Pharmacy of Peking University 2008, 70 (2008).

34. Crowe J.H., Crowe L.M.: Biochim. Biophys. Acta 939, 327 (1988). 